\title{
Atomic Absorption Spectrometry of Nanogram Amounts of Cadmium, Lead and Zinc after Precipitation with 8-Quinolinol
}

\author{
Kunihiko Akatsuka, Naoki Nobuyama and Ikuo Atsuya
}

Kitami Institute of Technology, Kitami 090

\begin{abstract}
A highly sensitive method was developed for the determination of cadmium, lead and zinc in natural waters by atomic absorption spectrometry after coprecipitation with 8-quinolinol. Quantitative recoveries of the metals were possible over the $\mathrm{pH}$ range $8-10$ when magnesium was present as a carrier ion. The precipitates thus formed were analyzed directly by an atomic absorption spectrometer equipped with a specially deviced graphite furnace and a miniature cup. Reliability of this procedure was confirmed by the determination of the metals in lake and river water samples. For the analysis of a $400-\mathrm{cm}^{3}$ portion of water sample, detection limits $\left(3 \sigma_{b}\right)$ for $\mathrm{Cd}(\mathrm{II}), \mathrm{Pb}(\mathrm{II})$ and $\mathrm{Zn}(\mathrm{II})$ are $0.6,2.3$ and $1.9 \mathrm{ng} \mathrm{dm}^{-3}$, respectively. A corresponding precision of $3-5 \%$ was typical.
\end{abstract}

Keywords 8-Quinolinol coprecipitation, magnesium carrier ion, lake water analysis, river water analysis, atomic absorption spectrometry, cadmium, lead, zinc

The concentrations of toxic trace elements in natural water are of considerable interest at present. In general, electro-thermal graphite furnace atomic absorption spectrometry (GFAAS) is the method of choice because of its high sensitivity for cadmium, lead and zinc. However, GFAAS is currently unable to determine directly cadmium and lead at the lower end of their reported concentration ranges in lake water ${ }^{1}$ or other natural waters. Therefore, an appropriate preconcentration is usually required.

8-Quinolinol (8-Q) is a well-characterized reagent and the nonselectivity of the reagent has made possible a wide range of analytical applications. Recently, complexation of trace elements with $8-Q$, followed by adsorption on activated carbon ${ }^{2}$ or $\mathrm{C}_{18}$ bonded silica $\mathrm{gel}^{3}$, has been developed. Although these techniques have been successfully employed in natural water analysis ${ }^{1,3-5}$ with GFAAS or ICP-AES, the final analyte specimen must be provided in the form of a liquid.

The coprecipitation method has been used for isolating trace elements from natural water, but GFAAS was until now limited to concentrates in solution. When the coprecipitation method is to be applied for isolation of trace elements, the precipitates must be submitted to analysis in the form of a liquid by dissolving the precipitates into appropriate acids. Redissolution involves several chemical stages in order to remove interfering matrix elements, thus decreasing the versatility of analysis and increasing the possibility of contamination.

We have already developed ${ }^{6-9}$ a solid sampling technique with AAS for the direct analysis of some powdered biological samples and coprecipitation samples. In the present study the technique has been explored for its usefulness in natural water analysis by coprecipitation of trace elements with 8-Q and the subsequent direct analysis of finely powdered precipitates with GFAAS. Simple, rapid and reliable analytical method for cadmium, lead and zinc with simultaneous multielement preconcentration was found to be possible for the lake water samples within the Japanese background level area.

\section{Experimental}

\section{Reagents}

Acids and ammonium hydroxide used were of the purest available reagent grade (Kanto Chemical Co.). Ultra clean water which was obtained from a Milli-Q purification system (Millipore Co.) was used. 8-Q (8hydroxyquinoline, reagent grade, Wako Pure Chemical Co.) was preliminarily purified, and then dissolved in hydrochloric acid, and a $2 \%(\mathrm{w} / \mathrm{v})$ solution was prepared. The purification procedure was as follows: 8-Q was dissolved in chloroform and the solution was shaken with an $0.05 \mathrm{~mol} \mathrm{dm}^{-3}$ EDTA aqueous solution at pH 10 in a Teflon separatory funnel. Then, the 
organic phase was separated and chloroform was evaporated. The solution of magnesium as a carrier metal ion, which was prepared by dissolving the pure metal (99.995\%) in hydrochloric acid, was also purified by the addition of $8-Q$ solution and a subsequent extraction with chloroform at $\mathrm{pH} 5$ to remove any extractable metal chelates.

Stock standard solutions of each element were prepared by dissolving the pure metals and diluting with water; working standards were obtained by serial dissolutions.

\section{Apparatus}

A Hitachi Zeeman atomic absorption spectrometer (Model Z-8000), equipped with a background compensation device, was used in combination with an electro-thermal graphite furnace of a cupped type and a miniature cup which was designed by us.6,7 A Hitachi AA data processor was employed for data handling.

A Mettler microbalance, model M-3 (readability $1 \mu \mathrm{g})$ was used. The maximum permissible weight for this balance is $2.9 \mathrm{~g}$.

\section{Coprecipitation procedure}

The coprecipitation procedure for the sample preparation was carried out in a simplified clean laboratory equipped with laminar flow benches, providing a class 10000 working environment.

In a standard procedure, an aliquot of sample solution $\left(100-400 \mathrm{~cm}^{3}\right)$ was placed in a Teflon beaker, into which $20 \mathrm{mg}$ of magnesium ions and $5 \mathrm{~cm}^{3}$ of the $8-\mathrm{Q}$ solution (100 $\mathrm{mg}$ as $8-\mathrm{Q}$ ) were added. This solution was adjusted to pH 9 by adding an aqueous ammonia solution, and allowed to age for $1 \mathrm{~h}$ at $70^{\circ} \mathrm{C}$ on a hot plate. It was then filtered by a glass filter (No. 4G). The filter was dried at $110^{\circ} \mathrm{C}$ for $1 \mathrm{~h}$ in a drying oven, and then weighed accurately. With a portion of the precipitate, the quantitative analysis was performed directly with AAS.

The powder standards used for calibration of absorbances were prepared by the standard procedure except that a $100-\mathrm{cm}^{3}$ portion of a standard solution containing various amounts of the respective metal ions was used. A blank powder standard was also prepared by the use of $100 \mathrm{~cm}^{3}$ of water for each sample preparation.

\section{Direct AAS measurement of precipitate}

The miniature cup was accurately weighed by use of the microbalance. After the precipitate was placed in the miniature cup, it was again weighed (difference $=$ net weight of the precipitate). The miniature cup was then inserted into the graphite furnace. The precipitate was dried, ashed and atomized according to the instrumental conditions shown in Table 1 .

For measurements of absorbance, at least five aliquots with different weights in the range $0.1-0.5 \mathrm{mg}$ were measured, and the absorbance value corresponding to $1 \mathrm{mg}$ of the precipitate was calculated. Oc-
Table 1 Instrumental conditions

\begin{tabular}{cccc}
\hline Element & $\mathrm{Cd}$ & $\mathrm{Pb}$ & $\mathrm{Zn}$ \\
\hline Wavelength/nm & 228.8 & 283.3 & $\begin{array}{c}213.8 \\
\text { (n) }\end{array}$ \\
Lamp current $/ \mathrm{mA}$ & 7.5 & 7.5 & 5.0 \\
Drying temp. $/{ }^{\circ} \mathrm{C}$ & - & 200 & 200 \\
(s) & & $(30)$ & $(30)$ \\
Ashing temp. $/{ }^{\circ} \mathrm{C}$ & 400 & 500 & 400 \\
(s) & $(30)$ & $(60)$ & $(30)$ \\
Atomization temp. $/{ }^{\circ} \mathrm{C}$ & 2500 & 2500 & 2500 \\
(s) & $(5)$ & $(5)$ & $(5)$ \\
\hline
\end{tabular}

a. This line was used for zinc recovery test.

casionally, to normalize the absorbances obtained in different concentration factors, we defined the total absorbances which were calculated from the absorbance obtained above multiplied by the total weight of precipitate obtained.

\section{Recovery studies}

The recoveries were calculated by analyzing the particular elements in the filtrates with ICP-AES (a Hitachi ICP atomic emission spectrometer, model 306 was used), where the original concentrations of the metals examined were $1 \mathrm{mg} \mathrm{dm}^{-3}$ (recovery test A). For preconcentration work in the submicrogram level (2$0.01 \mathrm{ppb}$ ), the recoveries were estimated by comparing the absorbance of the particular elements in the precipitate sample, which were divided by the concentration factor, to those of respective standard solution or NBS standard reference materials measured by the present AAS technique (recovery test B).

\section{Results and Discussion}

\section{Instrumental conditions for direct measurement of pre- cipitate samples}

Direct introduction of organic solvent extracts into the graphite furnace, despite reported success by some authors $^{10,11}$, has been unpopular. Difficulties have been reported in dispensing small volumes of organic solvents accurately. Additionally, the volatility of metal-organic compounds is reported to cause loss of the analyte, particularly in the presence of hydrocarbon halides, during drying and ashing steps of the furnace cycle. ${ }^{12,13}$ However, such difficulties were not encountered in a direct analysis of the powder sample by GFAAS.

The operating conditions for the furnace were investigated. The analyte specimen was prepared according to the standard procedure with magnesium carrier ions. As reported in the previous papers ${ }^{6,7}$, the 
drying step was not always necessary in the proposed solid sampling technique, but it was occasionally effective for ashing organic samples smoothly. Atomization temperatures were selected in view of the sensitivity. As a result, the temperature shown in Table 1 is recommended. The relative standard deviation (RSD) of signals evaluated by the peakheight data was obviously wrong, ranging from 5 to $15 \%$, whereas peak-area absorbance values gave reproducible results, the RSD of which was less than $5 \%$.

The effect of the ashing temperature was investigated in detail for each element. Parallel with this, the effect of the ashing time was also examined. The absorbances (peak area) became constant in the ashing temperature between 350 and $500^{\circ} \mathrm{C}$ for zinc, and 300 and $500^{\circ} \mathrm{C}$ for cadmium and lead. No volatility of the three elements in the analyte was observed at temperatures below $500^{\circ} \mathrm{C}$. The absorbances of the three elements were independent of the ashing time between $30 \mathrm{~s}$ and $120 \mathrm{~s}$ under the ashing temperature of $400-500^{\circ} \mathrm{C}$.

In order to ascertain the heating conditions, the absorption profiles for the elements were investigated. The profile for lead occasionally showed a two-peak pattern in the ashing conditions of $500^{\circ} \mathrm{C}$ for $30 \mathrm{~s}$ or lower than $400^{\circ} \mathrm{C}$ for $60 \mathrm{~s}$. However the absorption profiles for the three elements showed a symmetric pattern and no shoulder under the conditions shown in Table 1.

Although aluminum was less effective than magnesium as a carrier for quantitative recovery of the coprecipitation with 8-Q (described below), the heating conditions for the sample prepared with aluminum carrier ions were also examined. As a result, the conditions shown in Table 1 were recommended, except that an ashing temperature of $500^{\circ} \mathrm{C}$ is better for cadmium and zinc.

\section{Recovery efficiencies}

The effect of $\mathrm{pH}$ on the recoveries of cadmium(II), lead(II) and zinc(II) was investigated to find the conditions necessary for quantitative recovery from aqueous samples. We examined the recovery of the trace elements by using $8-\mathrm{Q}(100 \mathrm{mg})$ and a carrier ion as coprecipitant, precluding the use of thionalide and tannic acid because these reagents gave high blanks. ${ }^{14}$ The experiments were performed with $100 \mathrm{~cm}^{3}$ water containing magnesium or aluminum ions as carrier, according to the recovery test $\mathrm{A}$. The results are shown in Figs. 1 and 2. In Fig. 1, the pH range examined was limited to 6-11. Precipitation of magnesium(II) 8quinolinate did not take place significantly below pH 6 . As can be seen in Fig. 1, all of the three elements were quantitatively recovered in the presence of magnesium ions. However, aluminum ions were less effective than magnesium. As shown in Fig. 2, maximum recoveries of the elements were obtained at $\mathrm{pH} \mathrm{10-11,} \mathrm{but} \mathrm{the}$ recoveries were not $100 \%$. Cadmium, lead and zinc

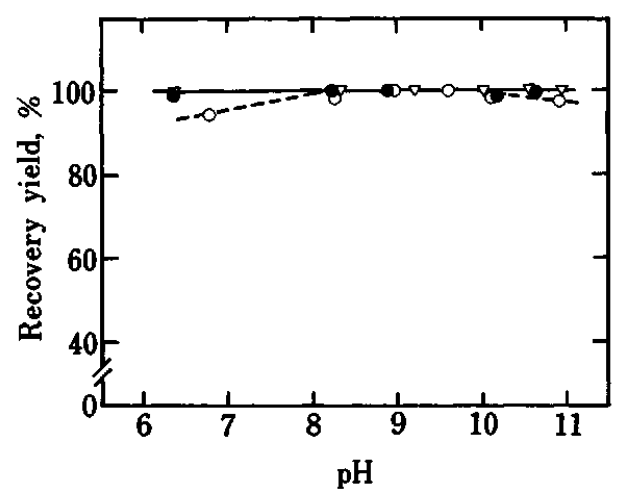

Fig. 1 Effect of pH on the coprecipitation of cadmium, lead and zinc with $8-Q$ in the presence of magnesium ions as a carrier. Metal ions taken, $100 \mu \mathrm{g}$; magnesium ions, $20 \mathrm{mg}$; $8-\mathrm{Q}, 100 \mathrm{mg}$; total aqueous volume, $100 \mathrm{~cm}^{3} ; \bullet, \mathrm{Cd}(\mathrm{II}) ; \mathrm{O}$, $\mathrm{Pb}(\mathrm{II}) ; \nabla, \mathrm{Zn}(\mathrm{II})$.

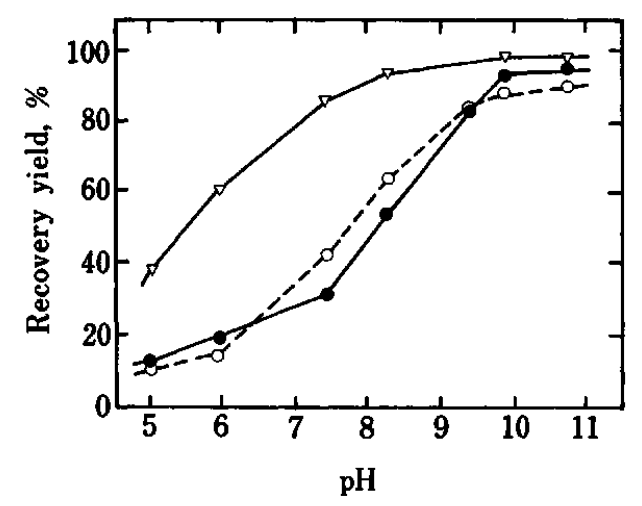

Fig. 2 Effect of $\mathrm{pH}$ on the coprecipitation of cadmium, lead and zinc with 8-Q in the presence of aluminum ions as a carrier. Metal ions taken, $100 \mu \mathrm{g}$; aluminum ions, $10 \mathrm{mg}$; $8-\mathrm{Q}, 100 \mathrm{mg}$; total aqueous volume, $100 \mathrm{~cm}^{3} ; \bullet, \mathrm{Cd}(\mathrm{II}) ; \mathrm{O}$, $\mathrm{Pb}(\mathrm{II}) ; \nabla, \mathrm{Zn}(\mathrm{II})$.

provided 96,92 and $98 \%$ recoveries, respectively, at $\mathrm{pH}$ 10.7 in the presence of $10 \mathrm{mg}$ of aluminum ions.

A further test of the recoveries for the metals (recovery test B) was carried out when known amounts of the metals in the order of submicrograms were spiked into $100 \mathrm{~cm}^{3}$ of pure water. Quantitative recoveries of all elements were found over the $\mathrm{pH}$ range $8-10$ with magnesium carrier ions. On the other hand, incomplete recoveries were confirmed with aluminum carrier ions. For cadmium, lead and zinc, 94, 84 and 96\% recoveries, respectively, were found at $\mathrm{pH} 10.5$ in the presence of $10 \mathrm{mg}$ of aluminum carrier ions. Consequently, in subsequent experiments, precipitates were prepared at $\mathrm{pH} 9$ in the presence of magnesium ions.

\section{Effect of 8- $Q$ amount and sample size}

Submicrogram amounts of the three elements were coprecipitated from $100 \mathrm{~cm}^{3}$ of water containing various amounts of $8-\mathrm{Q}$ up to $200 \mathrm{mg}$ and $20 \mathrm{mg}$ of 
Table 2 Detection limits, sensitivities and precision

\begin{tabular}{cccc}
\hline & $\begin{array}{c}\text { Concentration, } \\
\text { detection } \\
\text { limit }\left(400 \mathrm{~cm}^{3}\right)^{\mathrm{a}} / \\
\mathrm{ng} \mathrm{dm} \mathrm{m}^{-3}\end{array}$ & $\begin{array}{c}\text { Peak height } \\
\text { sensitivity } \\
(\mathrm{pg} / 0.0044 \mathrm{AU})\end{array}$ & $\begin{array}{c}\text { Precision }^{\mathrm{b}} \\
(\% \mathrm{RSD})\end{array}$ \\
\hline $\mathrm{Cd}$ & 0.6 & 2.3 & 4.2 \\
$\mathrm{Pd}$ & 2.3 & 50 & 3.3 \\
$\mathrm{Zn}$ & 1.9 & 1.2 & 4.5 \\
\hline
\end{tabular}

a. Defined as the concentration of analyte which gives a response equivalent to 3 times the standard deviation of the blank, based on a $400-\mathrm{cm}^{3}$ sample volume.

b. Expressed as a coefficient of variation of the analytical determination at $\mathbf{1 0}$-fold detection limits.

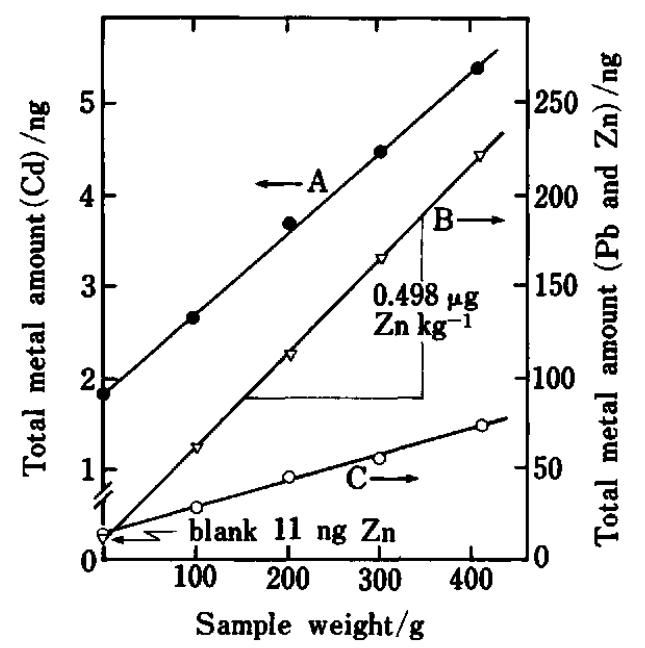

Fig. 3 Relationship between sample weight of river water sample and the total amount of metals in the final precipitate. A, cadmium; B, zinc; C, lead.

magnesium. As a result, the recoveries of the elements were independent of the amounts of $8-Q$ in the range $>40 \mathrm{mg}$.

Next, the standard method was applied to various volumes $\left(100-400 \mathrm{~cm}^{3}\right)$ of test solutions. The recovery yields of the elements were essentially independent of the sample volume (see Fig. 3). In this case (sample volume $400 \mathrm{~g}$ ), the concentration factor reached around 5500. Examinations on sample volumes larger than $500 \mathrm{~cm}^{3}$ were not made because high signals from reagent blanks were found due to large concentration factors $(>9000)$.

\section{Analytical figures of merits}

Detection limits, sensitivities and precision are given in Table 2. The sensitivity was calculated on the basis of the metal weight giving 0.0044 of peak-height absorbance. Detection limits have been estimated from the variation of blank values (i.e. $3 \sigma_{b}$ ). Precision is in
Table 3 Determination of $\mathrm{Cd}, \mathrm{Pd}$ and $\mathrm{Zn}$ in water of Lake Mashu (about $5 \mathrm{~m}$ depth)

\begin{tabular}{|c|c|c|c|}
\hline & \multicolumn{2}{|c|}{ Proposed method } & \multirow{2}{*}{$\begin{array}{l}\text { ICP-AES by } \\
\text { NIES } / \\
\mu \mathrm{g} \mathrm{dm}^{-3}\end{array}$} \\
\hline & $\mu \mathbf{g ~ k g}^{-1}$ & $\begin{array}{c}\text { Blank value / } \\
\text { ng }\end{array}$ & \\
\hline Cd & $0.0017 \pm 0.0004$ & $1.81 \pm 0.08$ & $<0.006$ \\
\hline $\mathrm{Pb}$ & $0.045 \pm 0.003$ & $11.9 \pm 0.3$ & $0.05 \pm 0.03$ \\
\hline $\mathrm{Zn}$ & $0.648 \pm 0.023$ & $10.7 \pm 0.3$ & $0.63 \pm 0.06$ \\
\hline
\end{tabular}

a. The values were reported by Nojiri et al. of the National Institute for Environmental Studies (Ref. 1).

the range $3-5 \%$ (RSD) at a level 10 -fold higher than the detection limits.

\section{Analysis of lake and river water}

The present method was applied to the determination of the three elements in river and lake water samples. The elements in the samples at sub-ppb level can be determined by the standard addition method using $100 \mathrm{~cm}^{3}$ of the sample waters. As blank values of the standard procedure were relatively high, the analysis was made by varying the sample weight in the range $100-400 \mathrm{~g}$. The concentration was calculated from the slope of the relationship between sample weight and total amounts of the elements. As an example, analytical results on a river water sample are shown in Fig. 3. The concentrations obtained were as follows: $\mathrm{Cd}, 0.0081 \pm 0.0002 \mathrm{ppb} ; \mathrm{Pb}, 0.145 \pm 0.004 \mathrm{ppb}$ and $\mathrm{Zn}, 0.498 \pm 0.010 \mathrm{ppb}$. The present result for $\mathrm{Cd}$ was in acceptable agreement with that obtained by Murozumi and Nakamura ${ }^{15}$ by isotope-dilution mass spectrometry for the same sample as that used in present study, i.e. $0.0107 \mathrm{ppb}$. The reference values for $\mathrm{Pb}$ and $\mathrm{Zn}$ in the river water have not been given yet.

Results for the water of Lake Mashu (about $5 \mathrm{~m}$ depth) are summarized in Table 3. The blank value listed in Table 3 was obtained from the Y-intercept of the relationship (c.f. Fig. 3), which was in good agreement with the blank value obtained by the standard procedure. In Table 3 , the present results are also compared with the values obtained by ICP emission spectrometry reported by Nojiri et al. ${ }^{1}$

Although the purification of the precipitants has not been investigated in detail, blanks can potentially be further lowered with use of cleaner precipitants. The advantages of the present method lie in its simplicity, good precision, high sensitivity and wide applicability range, since the solid sampling technique with AAS is simple and reproducible.

The authors wish to thank to Prof. Dr. M. Murozumi and Mr. S. Nakamura of Muroran Institute of Technology for their kindness in letting us use a part of their analytical results obtained on the river water sample by means of 
isotope-dilution mass spectrometry. We also thank Dr. Y. Nojiri, Dr. T. Kawai, Dr. A. Otsuki and Prof. Dr. Fuwa of the National Institute for Environmental Studies for their offer of the water of Lake Mashu, and Mr. Abe, machinery engineer of Kitami Institute of Technology, for his great efforts to make our miniature cups. This work was supported by a Grant-in-Aid for Scientific Research (No. 6147003) from the Ministry of Education of Japan, for which the authors are very grateful.

\section{References}

1. Y. Nojiri, T. Kawai, A. Otsuki and K. Fuwa, Water Res., 19, 508 (1985).

2. B. M. Vanderborght and R. E. V. Grieken, Anal. Chem., 49, 311 (1977)

3. H. Watanabe, K. Goto, S. Taguchi, J. W. McLaren, S. S. Berman and D. S. Russell, Anal. Chem., 53, 738 (1981).

4. R. E. Sturgeon, S. S. Berman, S. N. Willie and J. A. H. Desauliniers, Anal. Chem., 53, 2337 (1981).

5. S. N. Willie, R. E. Sturgeon and S. S. Berman, Anal.
Chim. Acta, 149, 59 (1983).

6. I. Atsuya and K. Itoh, Spectrochim. Acta, 38B, 1259 (1983).

7. I. Atsuya, K. Itoh, K. Akatsuka and K. Jin, Fresenius'Z. Anal. Chem., 326, 53 (1987).

8. K. Akatsuka and I. Atsuya, Anal. Chim. Acta, 202, 223 (1987).

9. K. Akatsuka and I. Atsuya, Fresenius' Z. Anal. Chem., 329, 453 (1987).

10. R. E. Sperling, Fresenius'Z. Anal. Chem., 292, 113 (1978); ibid., 299, 103 (1979).

11. R. P. Mitcham, Analyst [London], 105, 43 (1980).

12. G. Volland, G. Kolblin, P. Tschopel and G. Tölg, Fresenius' Z. Anal. Chem., 284, 1 (1977).

13. R. W. Dabeka, Anal. Chem., 51, 902 (1979).

14. E. E. Pickett and B. E. Hankins, Anal. Chem., 30, 47 (1958).

15. M. Murozumi and S. Nakamura, private communication (1986).

(Received February 22, 1988)

(Accepted April 13, 1988) 